\title{
Les systèmes produit-service comme business models pour l'économie circulaire : potentialités et
} limites

\section{Product-service systems as business models for the circular economy: potential and limitations}

\author{
Pereira Ángeles ${ }^{1}$, Vence Xavier ${ }^{2}$ \\ ${ }^{1}$ ICEDE. Universidade de Santiago de Compostela (USC), angeles.pereira@usc.es \\ ${ }^{2}$ ICEDE. Universidade de Santiago de Compostela (USC), xavier.vence@usc.es
}

RÉSUMÉ. L'économie circulaire (EC) définit un nouveau paradigme de production et de consommation qui cherche à réduire la consommation des ressources, à prolonger la durée de vie utile des produits et des services à travers diverses stratégies et boucles fermées, en essayant d'éviter autant que possible la génération de déchets ou en utilisant les déchets comme ressources pour de nouveaux processus. En tant que tels, les business models innovants et durables, y compris le Système-Produit-Service (SPS), sont considérés comme un élément central pour la réalisation de l'EC.

L'objectif de cet article est d'analyser la contribution des business models basés sur les SPS à la mise en œuvre de l'économie circulaire. II s'appuie sur un examen critique de la littérature de la fin des années 1980 à nos jours. Au cours des dernières années, les avantages potentiels du SPS pour améliorer l'éco-efficacité ont été soulignés. Toutefois, la contribution spécifique des SPS à l'économie circulaire reste à explorer. Bien que la littérature tend à plaider en faveur d'une plus grande efficacité des SPS axés sur les résultats, il existe en fait peu d'exemples. En revanche, l'économie circulaire ouvre de nouvelles opportunités pour la combinaison de produits et de services, dont la clé n'est pas tant la transition le long d'un axe produit-service que la réalisation de services de support pour les produits et leurs composants qui ont tendance à prolonger leur cycle de vie.

Dans notre article, nous soutenons que l'EC ouvre de nouvelles possibilités pour les services ou les combinaisons de produits et de services. Pourtant les SPS ont aussi des limites, qui concernent leur contribution en termes d'écoefficacité. Par conséquent, nous concluons qu'il est nécessaire d'adopter une perspective systémique et critique dans l'analyse des business models SPS et dans leur promotion afin qu'ils servent efficacement les principes de l'économie circulaire.

ABSTRACT. The circular economy (CE) defines a new paradigm of production and consumption that seeks to reduce resource consumption and to extend the useful life of products and services through various strategies and closed loops, by trying to avoid waste generation as much as possible or by using waste as a resource for new processes. As such, innovative business models, including product-service systems (PSS), are considered as a central element for the achievement of the CE.

This article analyzes PSS business models' contribution to the implementation of the circular economy. It is based on a critical review of the literature from the late 1980s up to the present day. In recent years, the potential benefits of PSS to improve eco-efficiency have been highlighted. However, the specific contribution of PSS to the CE remains to be explored. Although the literature tends to advocate for greater efficiency of results-oriented PSS, there are in reality only a few examples of this. By contrast, the CE opens up new opportunities for a combination of products and services. The key is not so much the transition along a product-service axis as it is the provision of support services for products and their components that prolong their life cycle.

In our paper, we argue that the CE opens up new possibilities for services or combinations of products and services. The PSSs that we analyzed, which are focused on unique cases and their contribution to eco-efficiency, have limits. Therefore, we conclude that it is necessary to adopt a systemic and critical perspective in the analysis of PSS business models and in their promotion so that they can effectively serve the principles of the circular economy.

MOTS-CLÉS. Economie Circulaire, Système-Produit-Service, business model, eco-efficacité, revue de la littérature.

KEYWORDS. circular economy, product-service systems, business model, eco-efficiency, literature review. 


\section{Introduction}

Les systèmes produit-service (SPS) incluent une diversité de modalités concrètes de relations fournisseur-client ayant en commun une certaine combinaison de produits et de services qui sont commercialisés ensemble (dans des proportions variables) afin de répondre aux besoins de l'utilisateur sans transférer la propriété du bien, mais en fournissant sa fonctionnalité pour une période donnée. Ces business models spécifiques peuvent être évalués sous de nombreux angles différents (valeur, fidélisation du client, stratégie de diversification, etc.), mais le potentiel de réduction des impacts environnementaux par rapport aux business models traditionnels est actuellement un sujet de première importance. En fait, les SPS sont souvent considérés comme modèles clés pour la transition vers l'économie circulaire. Au-delà d'une certaine littérature optimiste sur le potentiel de soutenabilité environnementale des SPS, une approche critique de leur impact réel est nécessaire.

La transition vers une économie circulaire (EC) est considérée comme une stratégie prometteuse pour le développement durable, en dissociant l'extraction des ressources matérielles et la consommation d'énergie de la croissance, tout en faisant une utilisation éco-efficace des ressources basée sur la récupération et le recyclage complet des matériaux utilisés et la transformation des déchets en nouvelles ressources [EMF 13] [GHI 14]. De plus en plus, l'attention est portée sur les changements nécessaires dans les business models et sur la configuration du système.

Une génération de propositions créatives pour de nouveaux modèles d'entreprise, des initiatives locales et des expériences revendiquant le concept d'économie circulaire a vu le jour. De nombreuses propositions et classifications différentes sont fournies par des travaux académiques et de consultants. Tout d'abord, une distinction fondamentale est établie entre les cycles biologiques et les cycles techniques [EMF 13]. Deuxièmement, en se concentrant principalement sur les cycles techniques des matériaux, on peut distinguer trois groupes différents de business models de l'économie circulaire ('Circular Economy Business Model'-CEBM) [STA 16]: a) ceux qui favorisent la réutilisation et prolongent la durée de vie utile par la réparation, la remise à neuf, la mise à jour, la récupération et la modernisation ; b) ceux qui transforment les biens anciens en ressources nouvelles par le recyclage des matériaux, et c) d'autres font appel à des changements dans la consommation : réduction de l'achat de biens (transfert du droit de propriété) en faveur de l'achat de la fonctionnalité ou de la consommation collaborative. Conformément à l'économie de fonctionnalité, certains CEBM se concentrent sur la dématérialisation de la consommation, en substituant des services/fonctions à la consommation matérielle.

Parmi les différents business models, ceux basés sur les systèmes produit-service (SPS) ont récemment été largement cités pour leur efficacité en termes de ressources et leur circularité [TUK 15]. La caractéristique fondamentale des SPS est de fournir la fonctionnalité d'un bien en tant que service sans systématiquement transférer la propriété du produit. Goedkoop et al [GOE 99] définissent un SPS simplement comme un ensemble commercialisable de produits et de services capables de répondre conjointement aux besoins d'un utilisateur; tandis que Mont [MON 02b : 239] suggère qu'un SPS est "un système de produits, de services, de réseaux et d'infrastructures de soutien qui est conçu pour être concurrentiel, satisfaire les besoins des clients et avoir un impact environnemental inférieur à celui des business models traditionnels". Au cours des dernières décennies, de plus en plus d'études se sont concentrées sur la contribution potentielle des SPS, entre autres choses, à l'efficacité des ressources. Cependant, la contribution des SPS à l'alignement sur les principes de l'économie circulaire n'est pas claire et prête à controverse [TUK 15].

D'un point de vue environnemental, on fait souvent valoir que le principal argument en faveur de systèmes produit-service considérés comme stratégiques pour la transition vers une économie plus 
économe en ressources réside dans les conséquences du maintien de la propriété des biens pour l'optimisation de leur utilisation et la responsabilité élargie des producteurs que ces modèles impliquent. Dans la mesure où le producteur conserve la propriété du produit, il est plus facile de retourner les produits usagés. Cette approche repose sur l'hypothèse que, dans un système où le fabricant ou le prestataire de services conserve la propriété du produit, il est encouragé à utiliser plus efficacement les ressources, à utiliser des produits et des technologies plus propres, à réduire les risques environnementaux associés au stockage, au transport, à la manutention et à l'élimination, à augmenter la récupération et le recyclage ou à consommer plus vert. Il serait encore plus incité à réduire l'obsolescence programmée et à prolonger la durée de vie des produits.

La plupart des études sur les SPS se sont concentrées sur leur capacité à améliorer l'éco-efficacité, mais pas exactement sur leur contribution aux principes de l'EC. Cette différence est importante car l'éco-efficacité, c'est-à-dire la réduction de l'utilisation des ressources par produit, n'affecte pas la vitesse du flux des produits et n'implique aucun cycle de service (par exemple, réparation). Cette stratégie peut être utilisée à la fois dans le modèle linéaire et circulaire et si la dimension temporelle n'est pas prise en compte, elle peut conduire à une augmentation des flux de ressources linéaires (par exemple, vendre plus d'unités d'un produit plus efficace) [BOC 16].

Cet article se concentre sur l'analyse de la contribution des différents systèmes produit-service à la réalisation de la circularité. L'objectif est d'élargir les connaissances sur la portée et les limites des SPS en tant que business models pour l'EC. Il s'agit d'une revue de la littérature, qui comprend les principales références sur la servitisation et les SPS depuis ses origines (fin des années 1980). L'analyse critique de la littérature empirique effectuée à la section 4 (tableau 3) repose sur une recherche par mots clés : SPS, éco-efficacité et Economie Circulaire à partir de la plateforme Web of Science, pour la période 2000-2017. A partir de la lecture des résumés, les articles dans lesquels la contribution du SPS à la durabilité environnementale et/ou à l'économie circulaire est explicitement analysée ont été sélectionnés. Au total, 14 articles ont été analysés.

L'article est structuré comme suit: dans la section suivante, nous analysons l'origine du SPS en tant que nouveau business model pour améliorer la compétitivité des entreprises; dans la section 3, nous passons en revue la littérature sur les potentialités du SPS en termes de bénéfices environnementaux par rapport aux autres modèles conventionnels basés sur les produits. Dans la section 4, nous présentons le débat sur les SPS en tant que business models susceptibles de contribuer aux objectifs de l'économie circulaire et discutons les résultats trouvés dans la littérature récente. Enfin, nous soulignons certaines idées clés dans la conclusion et présentons certaines implications pour la politique publique.

\section{Le SPS comme nouveau 'business model'}

Le concept de business model a été de plus en plus utilisé au cours des dernières années, dans différents domaines scientifiques. L'une des définitions les plus répandues d'un business model est fournie par Osterwalder et Pigneur [OST 10] [OST 05]. Un business model décrit la façon dont une organisation crée, distribue et saisit la valeur économique, sociale, etc. A cette fin, l'organisation est fondée sur un système de décisions et d'activités interconnectées et interdépendantes [BSI 17]. Sur la base de cette définition, certaines contributions ont été faites afin de parvenir à une définition d'un business model pour l'économie circulaire [VEN 19]. Par exemple, Linder \& Williander [LINDE 17] mettent l'accent sur la création de valeur à partir de l'utilisation de la valeur économique qui est conservée dans les produits après usage. En ce sens, le business model circulaire implique un retour des utilisateurs vers les producteurs et nécessite le développement d'activités telles que la réparation, la rénovation, le reconditionnement, ou le recyclage. Geissdoerfer et al. [GEI 17] conçoivent le business model circulaire comme un sous-type de business models durables, dont l'axe stratégique 
repose sur le bouclage, la réduction, le ralentissement, l'intensification et/ou la dématérialisation des cycles. Son objectif est de réduire au minimum les déchets et les émissions en dehors du système organisationnel et, par conséquent, d'améliorer les performances de durabilité. Les SPS en tant que business models sont justement basés sur la dématérialisation de la consommation en substituant le produit par les services.

Avant d'entrer dans l'analyse de la relation SPS-EC, il faut signaler que certains modèles de SPS sont assez anciens et leur développement est lié à des objectifs autres qu'environnementaux. Les premiers travaux sur la stratégie d'hybridation des produits et services par les entreprises manufacturières (servitisation) sont apparus dans le domaine du management et du marketing à la fin des années 1980. Comme le soulignent Vandermerwe \& Rada [VAN 88 : 315] "la 'servitisation' se produit dans presque toutes les industries à l'échelle mondiale. Sous l'effet de la déréglementation, de la technologie, de la mondialisation et de la pression concurrentielle féroce, les entreprises de services et les fabricants se lancent de plus en plus dans les services." La recherche sur la servitisation comme moyen d'améliorer la compétitivité des entreprises continue d'être un axe de recherche important dans ce domaine [BAI 07] [HUI 16] [KOH 18] [NEE 08] [RAB 16] [VIN 16] [VIN 13].

Oliva \& Kallenberg [OLI 03] justifient l'avantage pour les entreprises manufacturières d'ajouter des services à leur proposition de valeur pour leurs clients, en se fondant sur la possibilité d'obtenir des rendements supérieurs à partir d'une base installée de produits ayant un long cycle de vie, des marges plus élevées réalisées à partir des services et une plus grande stabilité des revenus, non assujettis aux cycles d'investissement et d'achat de matériel. De plus, étant plus difficiles à imiter, les services deviennent une source plus durable d'avantage concurrentiel. Dans le même ordre d'idées, la littérature la plus récente, qui ajoute même un potentiel environnemental, identifie un certain nombre d'avantages économiques, parmi lesquels [MON 02b] [ROT 07]: valoriser le produit, innover, améliorer les relations avec les consommateurs, anticiper la législation environnementale, diversifier l'offre, protéger le marché de l'entreprise des imitations, etc. Beuren et al. [BEU 13] ajoutent également aux avantages pour les fournisseurs, le potentiel d'innovation découlant du contrôle des produits et des services lors de leur utilisation ; la réduction des coûts et des ressources ; la maximisation des résultats ; la possibilité de vendre les connaissances acquises au cours du processus de développement en tant que services de conseil et de formation ou la réutilisation des produits en combinaison avec différents services. Aussi la littérature sur l'innovation dans les services ouverts [CHE 11] met en évidence la possibilité offerte par les services de sortir du "piège de l'indifférence" auquel conduit la production linéaire de biens de qualité homogène et à coûts réduits ; de même, ce lien stable entre producteur-client facilite la collaboration dans les processus d'innovation ouverte et l'appropriation des résultats de l'innovation.

Un aspect important des stratégies produit-service est qu'elles permettent d'établir une relation de type structurel avec le client, dans laquelle le fournisseur du produit-service devient une sorte de partenaire dans le processus de production. Cela se produit généralement de concert avec les stratégies d'impartition. "Cette stratégie fournit des services à la clientèle qui s'intègrent parfaitement à leur chaîne de valeur et à leur système de prestation de services, et tente ainsi d'attirer de nouveaux clients et de les fidéliser en créant une dépendance et des obstacles au changement. De plus, grâce à cette stratégie, les entreprises peuvent faire un meilleur usage de certaines compétences distinctives et réaliser des économies d'échelle" [FUR 10 : 716].

De toute évidence, l'intérêt d'une stratégie de SPS dépend d'un certain nombre de facteurs. Certains facteurs favorisent l'adoption de cette stratégie, par exemple, lorsque les entreprises sont en concurrence sur des marchés saturés et changeants, lorsque les produits sont complexes (besoin d'information et de formation pour le client), évoluent rapidement (besoin de mises à jour), sont 
radicalement novateurs (besoin pour les clients d'être sûrs de la valeur des produits), semblent durables (nécessitent des réparations et un entretien) ou doivent se distinguer d'autres produits. Sur la base de ces critères, Furrer [FUR 10] conclut que les différents types et sous-types de SPS ont des potentiels de rentabilité différents : a) il est plus intéressant pour une entreprise d'adopter des SPS " basés sur les produits " (le capital et les coûts resteront inchangés et ces SPS peuvent augmenter la valeur marchande pour l'utilisateur, la fidélité de la clientèle et aussi la vitesse de l'innovation) ; b) dans les modèles SPS " basés sur la performance ", le risque est plus grand pour le producteur, mais ils offrent aussi une plus grande valeur future (ils accélèrent l'innovation et la fidélisation de la clientèle); et c) les SPS " basés sur l'usage d'un produit " sont difficiles à évaluer par rapport à la capture de valeur par le fournisseur et, d'autre part, impliquent un abandon par les utilisateurs de valeur tangible et intangible.

Quoi qu'il en soit, les faits montrent que la diffusion des SPS semble beaucoup plus lente qu'on pourrait s'y attendre. Certains auteurs ont souligné une variété d'obstacles, y compris le fait que les avantages réalisés sont modestes, selon les cas [FUR 10] [NEE 08]. Toffel [TOF 02] identifie les coûts importants découlant de la modification de la relation contractuelle entraînée par le SPS (coûts de transaction, sélection adverse, aléa moral, risque financier, etc.). D'autres auteurs soulignent que sa diffusion dépend du stade de maturité de l'industrie, du cycle de vie du produit [VIN 15] ou des changements plus larges dans le contexte socio-technique, le comportement des clients, etc. [CES 13].

Les facteurs qui poussent les entreprises à adopter des systèmes produit-service peuvent être classés entre facteurs internes et externes [PER 16b]. Parmi les premiers, nous pouvons souligner : les économies financières et les revenus, l'utilisation accrue des actifs, l'accès aux sources secondaires de matières premières, la décision de la direction, l'évolution graduelle des activités existantes ou la détection d'une opportunité de marché spécifique et relativement petite comme complément à une activité existante, la réduction des risques et responsabilités ; l'influence indirecte de la possibilité de réaliser une amélioration environnementale a également été reconnue [BAR 03] [MON 02b] [WHI 99]. Les facteurs externes comprennent : l'influence des nouveaux développements réglementaires, notamment en matière de santé, de sécurité et d'environnement, les nouvelles opportunités de croissance dans les industries matures, la concurrence et la nécessité de protéger la part de marché ou la demande des clients eux-mêmes [BAR 03] [MON 02b].

La littérature analysée nous permet donc d'indiquer qu'à l'origine, la servitisation répond davantage à une stratégie de compétitivité des entreprises qu'à un business model proprement dit. Cependant, la mise en œuvre de SPS spécifique est comprise comme un nouveau business model, étant donné qu'il implique une nouvelle façon de créer, de saisir et de distribuer la valeur.

\section{Les SPS et leurs avantages potentiels pour l'environnement}

L'approche du SPS du point de vue de l'environnement et de la durabilité a été essentiellement développée à la fin des années 1990. Ce type de modèles suscite un intérêt croissant et, en particulier, il est proposé que la combinaison de produits et de services puisse contribuer au développement d'une économie fonctionnelle ou dématérialisée, réduire l'impact environnemental de l'activité économique et évoluer vers des modèles plus durables [GOE 99] [MON 02a] [STAH 97] [WHI 99]. L'idée de base de cette vision est que l'économie de fonctionnalité "optimise l'utilisation (ou la fonction) des biens et services et donc la gestion des richesses existantes (biens, connaissances et nature). L'objectif économique de l'économie de la fonctionnalité est de créer la valeur d'usage la plus élevée possible le plus longtemps possible tout en consommant le moins de ressources matérielles et d'énergie possible" [STAH 97]. 
White et al [WHI 99] suggèrent que trois situations économiques peuvent amener les fabricants à prendre des mesures pour réduire l'impact des produits au cours de leur cycle de vie:

-a) Lorsque le SPS entraîne l'internalisation des coûts d'utilisation ou d'élimination. Dans ce cas, le producteur serait incité à accroître l'efficacité de son produit en réduisant l'utilisation des ressources, en fabriquant ou en utilisant un produit plus durable nécessitant moins d'entretien ou en concevant un produit recyclable ou réutilisable. Toutefois, le potentiel dépend également de la valeur de ces coûts par rapport aux coûts totaux du produit et de la possibilité de répercuter ces coûts sur le client.

-b) Lorsque le produit a une valeur économique à la fin de son cycle de vie. Si le produit contient des composants réutilisables ou des matériaux précieux, de l'énergie, de la main-d'œuvre et du capital, le producteur peut trouver des incitations pour récupérer cette valeur par des activités de recyclage, de remise à neuf ou de réutilisation. De même, le potentiel de réduction dans ce cas serait limité par l'existence préalable ou non d'une infrastructure de valorisation ou par le coût de sa mise en place ex novo.

-c) Lorsque le produit devient une source de coûts plutôt que d'avantages. Le producteur ou le fournisseur s'efforce de réduire le nombre d'unités nécessaires par unité de service.

Cependant, il n'est pas si évident que la généralisation de la consommation de services ait une meilleure performance environnementale que celle des produits, comme nous le verrons plus loin. C'est pourquoi de nombreux auteurs affirment que l'essentiel est, d'une part, de concevoir les produits et services de manière à ce qu'ils aient un impact environnemental moindre au cours des différentes phases de leur cycle de vie complet : "Si les services doivent produire une économie plus verte, c'est parce qu'ils changent la façon dont les produits sont fabriqués, utilisés et éliminés - ou parce que, dans certains cas, ils supplantent complètement les produits physiques" [WHI 99: 10] ; et, d'autre part, au-delà des approches axées sur l'amélioration de la performance environnementale du côté de l'offre, une stratégie est nécessaire pour modifier également les modes de consommation, en cherchant à satisfaire les besoins des clients de manière durable (en ce sens, la philosophie " servicizing " selon laquelle les consommateurs ne sont pas intéressés à posséder des produits en soi, mais à leur utilité ou fonction, pourrait contribuer à une réduction des pressions sur le consommateur).

En tout état de cause, les conséquences environnementales du SPS ou de la servitisation ne sont pas aussi claires qu'on le suppose souvent et dépendent des types de SPS ou de la stratégie avec laquelle le système est conçu. En fait, certains auteurs proposent de différencier spécifiquement une catégorie de SPS durables [ROY 00], éco-services ou services durables [BAR 03] [HAL 14] [OMA 03]. Mont [MON 02a] affirme que l'un des principaux objectifs des systèmes produit-service devrait être de réduire l'impact environnemental de la consommation en fermant les boucles de matériaux, en diminuant la consommation grâce à des scénarios alternatifs d'utilisation des produits, en augmentant la productivité générale de l'utilisation des ressources et la dématérialisation du SPS et en fournissant des solutions système.

Tukker [TUK 04] réalise une estimation approximative des avantages environnementaux qui peuvent être obtenus par les différents sous-types de SPS, par rapport aux business models de référence basés sur les produits. Il indique donc que les services liés aux produits, les services de conseil et de consultation et les types de SPS fondés sur des systèmes de location ou d'utilisation partagée sont susceptibles d'avoir des avantages environnementaux marginaux. Dans le cas des systèmes de location, de partage ou d'utilisation collective, les avantages seront plus importants si les impacts sont davantage liés à la phase de production du bien qu'à la phase d'utilisation. L'externalisation ou la gestion des activités permettra d'obtenir de meilleurs résultats si les gains 
d'efficacité sont liés aux matériaux et aux artefacts plutôt qu'aux ressources humaines. Les SPS basés sur le paiement à l'utilisation peuvent conduire à des améliorations progressives mais non radicales s'il n'y a pas de changement dans le système technologique. Enfin, les systèmes fonctionnels axés sur les résultats offrent une plus grande probabilité d'obtenir des avantages environnementaux puisque le producteur a plus de liberté pour concevoir un système à faible impact. Le tableau 1 ci-dessous résume graphiquement ces conclusions (tableau 1).

\begin{tabular}{|c|c|c|c|c|c|}
\hline \multirow[t]{2}{*}{ Type de SPS } & \multicolumn{5}{|c|}{ Impacts par rapport à la situation de référence (produit) } \\
\hline & Pire & Égale & $\begin{array}{l}\text { Réduction progressive } \\
(<20 \%)\end{array}$ & $\begin{array}{l}\text { Réduction considérable } \\
(<50 \%)\end{array}$ & $\begin{array}{l}\text { Réduction radicale } \\
(<90 \%)\end{array}$ \\
\hline Service lié au produit & & $4-$ & $\rightarrow$ & & \\
\hline Conseil et consultance & & $4--$ & $\rightarrow$ & & \\
\hline Location de produits & & --- & --- & & \\
\hline $\begin{array}{c}\text { Location et partage de } \\
\text { produits }\end{array}$ & & & & & \\
\hline $\begin{array}{l}\text { Mise en commun de } \\
\text { produits }\end{array}$ & & $4-$ & $-----------\rightarrow$ & & \\
\hline Gestion de l'activité & & $4-$ & $-------\rightarrow$ & & \\
\hline $\begin{array}{c}\text { Paiement à l'unité } \\
\text { d'utilisation }\end{array}$ & & $<$ & $----------\rightarrow$ & & \\
\hline Résultat fonctionnel & & $4-$ & ---------------- & --------- & \\
\hline
\end{tabular}

Tableau 1. Caractéristiques de durabilité (environnementale) des sous-types du SPS [TUK 04]

Notes:

Location, partage: radicalement meilleur si l'impact est lié à la production du produit.

Mise en commun: réductions supplémentaires par rapport au partage / location si impacts liés à la phase d'utilisation.

Location, partage, mise en commun : encore plus si le système conduit à un comportement de nonutilisation.

En appliquant différents mécanismes ou stratégies, chaque sous-type de SPS a le potentiel de réaliser différentes améliorations environnementales par rapport aux business models traditionnels. Le tableau 2 résume les mécanismes d'entretien écologique identifiés par l'Environmental Protection Agency des États-Unis [EPA 09]. 


\begin{tabular}{|c|c|c|}
\hline Type de SPS & $\begin{array}{l}\text { "Mécanisme d'éco-servicisation " (Comment } \\
\text { les SPS innovateurs / émergents de cette } \\
\text { catégorie peuvent-ils réaliser des gains } \\
\text { d'éco-efficacité par rapport au statu quo?) }\end{array}$ & $\begin{array}{l}\text { Potentiel d'amélioration de } \\
\text { l'environnement }\end{array}$ \\
\hline \multirow{3}{*}{$\begin{array}{l}\text { Services liés aux } \\
\text { produits }\end{array}$} & Reprise du produit en fin de vie & $\begin{array}{l}\text { Augmentation de la réutilisation et de la } \\
\text { récupération des matériaux. }\end{array}$ \\
\hline & & $\begin{array}{l}\text { Réduction de la demande de matières } \\
\text { vierges et des impacts sur l'élimination. }\end{array}$ \\
\hline & Entreprises de recyclage et de ré usinage & $\begin{array}{l}\text { Réduction du nombre de produits au fil du } \\
\text { temps; réduction des impacts au cours des } \\
\text { phases précédant l'utilisation et des } \\
\text { impacts de l'élimination. }\end{array}$ \\
\hline $\begin{array}{l}\text { Conseil en matière de } \\
\text { produits }\end{array}$ & Prolongement de la durée de vie du produit & $\begin{array}{l}\text { Réduction des impacts du produit en cours } \\
\text { d'utilisation et prolongation de la durée de } \\
\text { vie du produit. }\end{array}$ \\
\hline Location du produit & Conseil en efficacité & $\begin{array}{l}\text { Réduction des charges environnementales } \\
\text { avant la phase d'utilisation, élimination } \\
\text { appropriée et/ou récupération améliorée. }\end{array}$ \\
\hline $\begin{array}{l}\text { Utilisation conjointe } \\
\text { (séquentielle et mise } \\
\text { en commun) }\end{array}$ & $\begin{array}{l}\text { Approches de conception écologique, créant } \\
\text { des produits plus durables avec des proportions } \\
\text { accrues de pièces recyclables / réutilisables. }\end{array}$ & $\begin{array}{l}\text { Réduction du nombre total de produits } \\
\text { requis ; réduction des impacts au cours } \\
\text { des phases précédant l'utilisation et des } \\
\text { impacts de l'élimination. }\end{array}$ \\
\hline $\begin{array}{l}\text { Unité de paiement à } \\
\text { l'acte }\end{array}$ & & $\begin{array}{l}\text { Comportements de conservation, réduisant } \\
\text { l'utilisation du produit et les impacts } \\
\text { environnementaux liés à la phase } \\
\text { d'utilisation. }\end{array}$ \\
\hline $\begin{array}{l}\text { Gestion de l'activité / } \\
\text { externalisation }\end{array}$ & $\begin{array}{l}\text { Signal de prix basé sur l'utilisation à } \\
\text { l'utilisateur }\end{array}$ & $\begin{array}{l}\text { Réduction de l'intensité matérielle et } \\
\text { énergétique; }\end{array}$ \\
\hline & $\begin{array}{l}\text { Réduction des coûts et gains d'efficacité dans } \\
\text { les intrants de matières et d'énergie }\end{array}$ & Réduction de la production de déchets, de \\
\hline Résultats fonctionnels & Dématérialisation informatique & $\begin{array}{l}\text { l'utilisation d'énergie ou de la } \\
\text { consommation d'autres biens ou services } \\
\text { problématiques. }\end{array}$ \\
\hline
\end{tabular}

Tableau 2. Mécanismes d'écologisation des services et amélioration potentielle de l'environnement [EPA 09]

De la même manière que le potentiel d'amélioration environnementale du SPS est généralement reconnu, de nombreux auteurs préviennent également que cette amélioration n'est pas garantie et que les avantages potentiels dépendent dans une large mesure du type de SPS analysé [TUK 04] [TUK 06] [VAN 13]. En général, les systèmes axés sur les produits ajoutent simplement des services à un produit, de sorte que l'amélioration environnementale se limite à un meilleur entretien ou à une plus grande recyclabilité. Les améliorations des SPS axés sur l'utilisation peuvent provenir d'une 
utilisation plus intensive du même produit; toutefois, lorsqu'il s'agit de prolonger la vie d'un produit moins efficace, les améliorations peuvent être limitées. Les SPS axés sur les résultats sont ceux qui, compte tenu de la liberté du producteur de remplir une fonction ou de satisfaire une demande, ont le plus grand potentiel de réduction de l'impact environnemental.

En outre, il est important de reconnaître l'existence d'"effets rebond", c'est-à-dire d'effets secondaires négatifs pour l'environnement, résultant d'une transition vers le service, tels qu'une augmentation de la demande résultant d'une baisse des prix, ou un comportement moins responsable de la part du consommateur ; c'est à dire, dans la mesure qu'il n'est pas propriétaire il pourrait réduire les incitations à un responsable usage-maintenance des biens (aléa moral). Nous devons également ajouter que la mise en œuvre des modèles de services, même s'ils ont des impacts environnementaux moindres, se heurte à de nombreux obstacles qui rendent difficile leur diffusion à grande échelle. En ce sens, dans un travail précédent relatif à l'agriculture [PER 17] nous avons identifié l'existence d'obstacles internes tant de la part des agriculteurs-clients que de la part de entreprises, ainsi qu'en raison des caractéristiques spécifiques de chaque culture, du terrain et aussi climatologique, pour permettre une large diffusion des services de protection phytosanitaires des végétaux fondés sur les principes de la lutte intégrée contre les ravageurs. Dans le même esprit, le projet européen SPREE a mis en évidence différents résultats qui peuvent être obtenus par différents modèles de SPS dans différents secteurs (mobilité, agriculture et eau) et dans différents pays, dans leur contribution au découplage de la croissance économique des impacts environnementaux. Dans la section suivante, nous discuterons de la relation spécifique entre SPS et EC, en nous fondant sur la littérature empirique récente.

\section{Nouvelles opportunités et limites des SPS dans l'Économie Circulaire}

L'analyse de la relation entre EC et SPS est toutefois récente. Une première analyse bibliométrique en ce sens a été réalisée par Michelini et al [MIC 17], qui traite du rôle du SPS dans l'atteinte des objectifs d'efficacité des ressources par l'entremise de l'EC. Ils soulignent que les études concluent souvent que seuls les SPS axés sur les résultats contribuent réellement à l'efficacité des ressources et à la circularité. De notre point de vue, cet article n'est pas concluant, car l'analyse se limite à des cas particuliers de SPS. Une vision systémique et la combinaison de différents modèles économiques tout au long de la vie d'un produit ou service, tels que ceux exigés par l'économie circulaire serait nécessaire [ANT 16] [URB 17].

Un autre étude récente [YAN 18], à partir d'une seule étude de cas, analyse la création de valeur de différents types de SPS pour la circularité et conclut que ces business models peuvent créer de la valeur circulaire grâce à différentes stratégies : bouclages internes de réutilisation et de récupération, bouclages plus longs et utilisation en cascade en tirant parti des co-produits et sous-produits dans d'autres chaînes de valeur. Bref, si tout type de SPS semble augmenter le taux de réparation, de réutilisation, de remise à neuf et de recyclage, les plus prometteurs sont axés sur l'utilisation et, surtout, les résultats. Toutefois, le SPS comporte des limites pour motiver la remise à neuf et le recyclage, puisque l'entreprise peut ne pas savoir comment identifier et tirer parti de la valeur contenue dans les matériaux à la fin de leur vie utile; ou cette valeur peut également être beaucoup plus faible que le coût de la remise à neuf et du recyclage.

Contrairement à la vision traditionnelle du SPS en tant que transition du produit aux services avec des gains d'éco-efficacité supposés liés à la responsabilité élargie des producteurs, l'économie circulaire apporte de nouveaux défis et de nouvelles opportunités pour le SPS. Allant plus loin que les objectifs d'éco-efficacité, l'économie circulaire exige des business models qui contribuent à obtenir la valeur d'usage maximale des produits sur la plus longue période et à concevoir les déchets comme ressources. Par exemple, la Fondation Ellen MacArthur (EMF, 2013) a créé le cadre 
d'analyse ReSOLVE (REgenerate, Share, Optimise, Loop, Virtualise, Exchange) pour expliciter les business models qui pourraient contribuer à l'économie circulaire. Plusieurs font partie de la famille SPS. Temporairement, nous pouvons dire que les SPS basés sur les produits et basés sur l'usage des produits peuvent privilégier les stratégies de partage "Share" (partage d'actifs, réutilisation et seconde main, prolongation de la durée de vie par la maintenance, conception pour la durabilité, mise à niveau, etc.) et d'"Optimisation" (amélioration de l'efficacité des produits, élimination des déchets dans la chaîne d'approvisionnement, automatisation, technologies de télédétection et de contrôle à distance). D'autres SPS, tels que ceux axés sur la performance, encourageant l'adoption des stratégies de bouclage "Loop" (refabrication de produits et composants, recyclage des matériaux), d'utilisation des technologies de l'information "Virtualise" (services électroniques) et même de remplacement "Exchange" (remplacement des matériaux non renouvelables par de nouvelles technologies, comme l'impression 3D).

Comme indiqué plus haut, le débat sur la contribution des SPS à l'économie circulaire n'en est qu'à ses débuts. Spring \& Araujo [SPR 17], sur la base du concept de "biographie du produit", affirment que les possibilités des SPS dans le contexte de l'EC s'élargissent : la transition vers le service n'est pas un mouvement à sens unique le long d'un axe. Les produits sont réparés, renouvelés, mis à jour, désassemblés, réassemblés et seulement éliminés définitivement. Cette approche implique la création de nouvelles relations de réseau autour de diverses activités telles que la logistique inverse, le démontage des produits, le tri des composants des produits, le recyclage des matériaux, etc. Par conséquent, les possibilités d'affaires du point de vue de l'EC vont au-delà de la réparation et de la restauration et font intervenir des chaînes de valeur en amont et en aval.

Ce rôle élargi des services dans la transition vers l'économie circulaire est aussi souligné dans l'approche de la "colline de valeur" ('value hill') [ $\mathrm{ACH}$ 16]. Les stratégies visant à prolonger la durée de vie des produits vont de pair avec des stratégies de croissance axées sur les services. Les services peuvent contribuer à une meilleure performance des produits dans trois domaines clé: a) la conception des processus : services autour de processus qui augmentent le potentiel de réutilisation et la recyclabilité des produits industriels et autres, sous-produits et flux de déchets ; b) la gestion des valeurs : services autour de la gestion des informations, matériaux, transparence, paiements et gouvernance dans un réseau circulaire; c) le traçage : services pour faciliter le trading et la commercialisation des matières premières secondaires.

Conformément à ces propositions antérieures, nous devrions reconnaître que les SPS axés sur les produits peuvent apporter une contribution importante à la réalisation de l'EC, si l'on adopte la perspective systémique nécessaire.

La relation ambigüe entre les SPS et l'économie circulaire découle précisément de l'existence d'une diversité de modèles de SPS, qui répondent à une nature et à des objectifs qui sont également très divers. Afin de discuter de la relation entre les SPS et l'économie circulaire, nous devons prendre en compte le fait que certaines formes de SPS existent depuis longtemps, bien avant leur mise en relation avec l'EC. Ainsi, les SPS ont souvent consisté en un nouveau business model visant à créer de la valeur pour l'entreprise dans le cadre d'une nouvelle relation avec les clients, par exemple, une relation client plus intense et durable au lieu d'une vente unique. Dans certains cas, les objectifs de l'entreprise sont axés sur la satisfaction des besoins d'un client particulier, tandis que dans d'autres cas, l'objectif est de créer un flux continu de revenus pour la prestation de services plutôt qu'un revenu unique provenant de la vente d'un bien. L'objectif est de contrôler l'ensemble de la chaîne de valeur jusqu'au consommateur afin de stabiliser les marchés à long terme, de lier les clients et d'augmenter les marges [OLI 03] [REI 14] [VAN 88]. 
Pour cette raison, notre but est de contribuer à l'étude du potentiel et des limites du SPS pour la réalisation des principes de l'économie circulaire. Pour réaliser cette analyse, nous partons des différents SPS analysés dans la littérature et analysons dans quelle mesure la création de valeur est circulaire, en nous basant sur la classification suivante des stratégies circulaires [BOC 16] [MER 18] :

a. Ralentissement des boucles de ressources ( $\mathrm{R}$ dans le tableau suivant): il s'agit d'identifier si les SPS analysés dans la littérature, basés sur la conception de produits durables et l'allongement de la durée de vie du produit, augmentent ou intensifient la durée d'utilisation des produits, contribuant ainsi au ralentissement des flux de ressources :

-i) Modèles d'accès et de performance : répondre aux besoins sans transférer la propriété du produit ;

-ii) Extension de la valeur du produit : pratiques de re-manufacture et de rénovation ;

-iii) Modèle classique durable : conception de produits durables (durabilité et réparation);

-iv) Favoriser la suffisance : prolonger la durée de vie du produit par la durabilité, la mise à niveau, la réparation et les garanties ;

b. Fermeture des boucles de ressources ( $\mathrm{F}$ dans le tableau suivant) : SPS qui induisent des stratégies basées sur le recyclage, fermant le cycle entre la post-utilisation et la production.

-v) Extension de la valeur de la ressource : transformation des déchets en une ressource précieuse ;

-vi) Symbiose industrielle : les sous-produits d'un procédé industriel deviennent des intrants pour une autre industrie.

Les principaux résultats des articles examinés sont résumés au tableau 3 et analysés ci-dessous. 
(Komoto, Tomiyama,

Nagel, Silvester, \&

Brezet, 2005)

[KOM 05]

\section{(Evans, Partidário, \& \\ Lambert, 2007)}

[EVA 07]

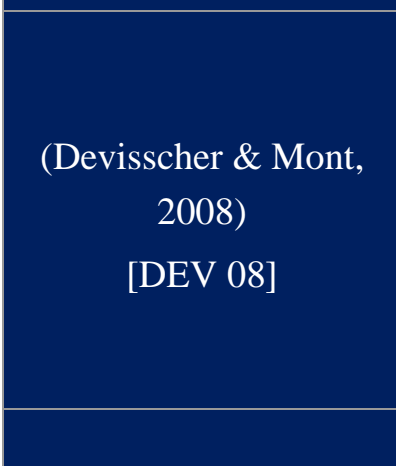

(Lelah, Mathieux, \& Brissaud, 2011)

[LEL 11]

\section{(Lee, Geum, Lee, \&}

Park, 2012)

[LEE 12]

(Lindahl, Sundin, \& Sakao, 2014)

[LIN 14]

Modèles alternatifs de la fonction de lavage

(événements SPS):

Traditionnel avec des services d'entretien,

de mise à niveau et de remise à neuf;

Ventes fonctionnelles: modèle de paiement

à l'utilisation;

Modèle de partage: service de commodité;

Modèle professionnel : service de

blanchisserie

Services de livraison de repas pour les

personnes âgées et les employés des PME

Amélioration de la logistique;

Planification des commandes d'aliments

Cencoop. Coopérative qui fournit des Installations à petite échelle et installations de

services aux producteurs de café

transport partagées

Utilisation partagée de l'équipement, nettoyage,

réparation et entretien

Système de vermicompostage pour déchets ;

commerciale,

Réutilisation des modules

(R) Modèle d'accès; Extension de la valeur du produit

(R) Modèle d'accès - aucune information disponible concernant la valeur $\mathrm{CE}$

(R) Modèle d'accès; Extension de la valeur du produit

(R) Modèle d'accès; Extension de la valeur du produit

(F) Extension de la valeur des ressources

Réseau machine-to-machine pour la

collecte d'informations dans les circuits de

collecte des déchets de verre

Optimisation de la collection;

Services techniques: installation, maintenance,

exploitation et mise à niveau du réseau de capteurs

Mutualisation des infrastructures

Part de la bicyclette dans le transport

Système de vélos publics (de Séoul)

1) Bouchons de mandrins pour papeteries

accès, entretien et reprise
Réutilisation et recyclage des matériaux (près de $100 \%$ )
(R) Modèle d'accès; Extension de la valeur du produit

(R) Modèle d'accès - aucune information disponible concernant la valeur $\mathrm{CE}$

(R) Modèle de performance; Extension de la valeur du produit;

(F) Extension de la valeur des ressources 


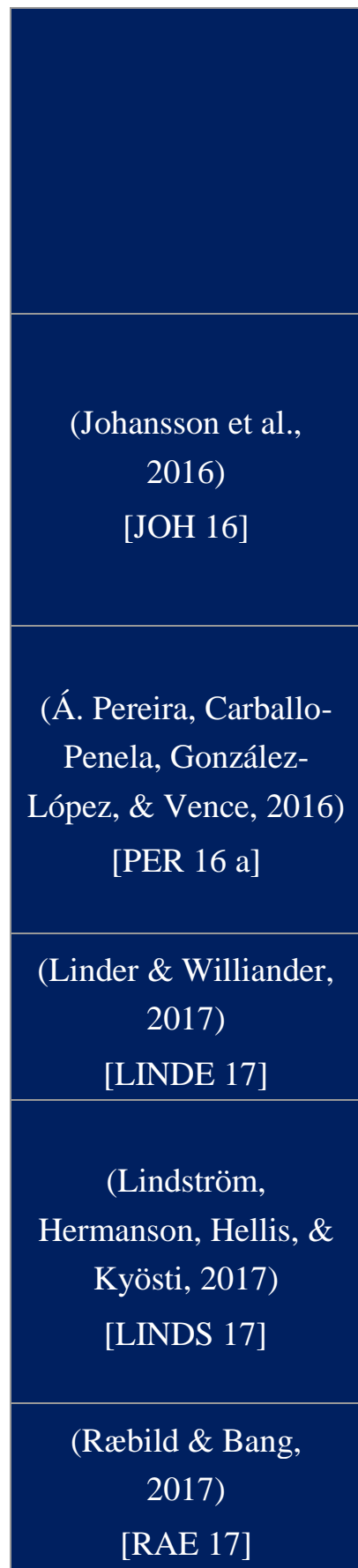

2) Nettoyage de l'extérieur des bâtiments : solutions de nettoyage

3) Services de compacteurs de sol
2) Méthode de lavage sans détergents et une planification et une opération holistique

3) Location de compacteurs de sol ; durée de vie prolongée grâce à la conception, à l'entretien et à la remise à neuf.
(R) Modèle de performance

(R) Modèle d'accès; Extension de la valeur du produit; Modèle classique longue durée de vie
Solutions minières urbaines: mise à

disposition de capacités spécialisées dans des services spécifiques:

Services de machinerie spécialisée pour atteindre les spaces restreints et détecter les métaux précieux;

Comment réutiliser les matériaux (p. ex.

béton)

Services coopératifs de machinerie agricole, d'élevage de génisses et de production fourragère

\section{Offre de vélos électriques PS : modèle} d'abonnement

Une PME de haute technologie fournit un service de recyclage aux municipalités et aux entreprises qui sont responsables de la gestion des conteneurs de recyclage où principalement les ménages déposent du papier, des matériaux d'emballage, des métaux, du verre et des plastiques, etc.

Service d'abonnement pour les vêtements de bébé
Exploration de nouvelles opportunités SPS : un marché secondaire plus actif et prolongé avec des solutions de maintenance proactives grâce aux capacités de télématique et de data mining.

Modèle de partage : équipements et installations

Modèle de partage : planification, équipement, installations et information

Modèle de partage : planification, équipement et information

Remise à neuf et réutilisation

(R) Modèle d'accès; Extension de la valeur du produit

(R) Modèle d'accès; Extension de la valeur du produit

(R) Modèle de performance;

(F) Symbiose industrielle (purins)

(R) Modèle de performance; Extension de la valeur du produit

(R) Modèle d'accès; Extension de la valeur du produit

Service d'information qui augmente l'efficacité de la gestion du recyclage en optimisant la vidange des contenants au fur et à mesure qu'ils sont remplis à pleine capacité, afin d'éviter les déchets lorsqu'ils sont surchargés et de ne pas vider les contenants qui ne doivent pas encore l'être (évitant ainsi les kilomètres non nécessaires)

Réutilisation circulaire des produits : conception pour la durabilité
(R) Modèle d'accès - soutient

l'économie circulaire, mais il ne s'agit pas d'un modèle d'activité de base pour $\mathrm{CE}$

(R) Modèle d'accès; Modèle classique à longue durée de vie 


\begin{tabular}{|c|c|c|c|}
\hline \multirow{3}{*}{$\begin{array}{l}\text { (Stål \& Jansson, 2017) } \\
\text { [STAL 17] }\end{array}$} & \multirow[t]{3}{*}{$\begin{array}{l}9 \text { entreprises de mode suédoises mettant en } \\
\text { œuvre } 3 \text { SPS différents }\end{array}$} & Le crédit-bail (usage-SPS) ; & $\begin{array}{l}\text { (R) Modèle d'accès; Extension de la } \\
\text { valeur du produit }\end{array}$ \\
\hline & & $\begin{array}{l}\text { Conseils pour le kit de lavage et de réparation } \\
\text { (produit-SPS); }\end{array}$ & (R) Favoriser la suffisance \\
\hline & & Système de reprise (produit-SPS) & $\begin{array}{l}\text { (F) Extension de la valeur des } \\
\text { ressources }\end{array}$ \\
\hline \multirow{2}{*}{$\begin{array}{c}\text { (Bressanelli, } \\
\text { Adrodegari, Perona, \& } \\
\text { Saccani, 2018) } \\
\text { [BRE 18] }\end{array}$} & \multirow[t]{2}{*}{$\begin{array}{l}\text { SPS basé sur l'utilisation d'appareils } \\
\text { électroménagers }\end{array}$} & Abonnement : modèle d'accès et contrat de reprise & \multirow[b]{2}{*}{$\begin{array}{l}\text { (R) Modèle d'accès; Extension de la } \\
\text { valeur du produit; } \\
\text { (F) Extension de la valeur des } \\
\text { ressources }\end{array}$} \\
\hline & & $\begin{array}{l}\text { Prolongement de la durée de vie : garanties } \\
\text { supplémentaires, maintenance prédictive, réparation, } \\
\text { conception pour durer, mise à niveau. }\end{array}$ & \\
\hline \multirow{3}{*}{$\begin{array}{c}\text { (Yang, Smart, Kumar, } \\
\text { Jolly, \& Evans, 2018) } \\
\text { [YAN 18] }\end{array}$} & $\begin{array}{l}\text { Services techniques ajoutés à la vente } \\
\text { d'unités de produits de séparation d'air }\end{array}$ & $\begin{array}{l}\text { Entretien et réparation réguliers des produits pendant } \\
\text { la phase d'utilisation }\end{array}$ & (R) Favoriser la suffisance \\
\hline & Leasing d'unités de séparation d'air & $\begin{array}{l}\text { Réutilisation, récupération, remise à neuf et } \\
\text { recyclage des générateurs de gaz }\end{array}$ & $\begin{array}{l}\text { (R) Extension de la valeur du produit; } \\
\text { Intensification }\end{array}$ \\
\hline & Vente de gaz industriels & $\begin{array}{l}\text { Réutilisation, récupération, remise à neuf et } \\
\text { recyclage des générateurs de gaz et réutilisation des } \\
\text { co-produits et sous-produits (gaz) }\end{array}$ & $\begin{array}{l}\text { (R) Extension de la valeur du produit; } \\
\text { (F) Symbiose industrielle }\end{array}$ \\
\hline
\end{tabular}

(R) - Ralentissement des boucles des ressources (F) - Fermeture des boucles des ressources

Tableau 1. Contribution des SPS à la création de valeur circulaire 
Un premier aspect que l'on peut souligner est la rareté ou le lien encore très limité entre le SPS et l'EC. Seuls les articles publiés après 2016 font explicitement référence à l'économie circulaire. En outre, cela se fait dans plusieurs cas par opportunité, c'est-à-dire que certains des articles ne mentionnent l'économie circulaire que dans le résumé ou dans l'introduction, mais il n'y a pas d'autre analyse de la relation entre les deux concepts dans le texte (voir par exemple, Johansson et al. [JOH 16]; Stål \& Jansson [STAL 17]).

Le deuxième aspect qui ressort clairement des articles analysés est que la plupart des SPS sont axés sur les produits ou l'utilisation. Seulement quelques études de cas concrets analysent les SPS axés sur les résultats, comme Lindahl et al [LIN 14] et Yang et al [YAN 18]. Tout au long des cas analysés, nous observons que la plupart des SPS appliquent des stratégies d'EC liées au " ralentissement de la boucle des ressources ". Logiquement, les fournisseurs de SPS axés sur l'utilisation, que ce soit par le biais de systèmes d'utilisation partagée, de leasing ou de renting, contribuent à l'EC par une utilisation plus efficace (intensive) du même produit. En outre, ces systèmes facilitent ou impliquent généralement l'application de stratégies visant à accroître la valeur $\mathrm{du}$ produit. Cela se produit principalement lorsque le fabricant ou le fournisseur de services conserve la propriété du produit. Dans de tels cas, l'intérêt du propriétaire est l'entretien, la réparation, la mise à jour, le renouvellement et même la refabrication des produits. Dans certains cas, l'importance de la conception pour faciliter la réparabilité, la rénovation et la refabrication est soulignée, comme dans des articles de Lindahl et al [LIN 14] Raebild \& Bang [RAE 17] ou Bresanelli et al [BRE 18].

Les SPS axés sur l'usage se concentrent principalement sur les actifs qui sont souvent très coûteux, ou sur les technologies de l'information qui exigent des connaissances et des compétences particulières [LEL 11] [LINDS 17]. Pour cette raison, il s'agit de SPS qui favorisent la conservation de la propriété par les fabricants ou, le cas échéant, par un intermédiaire de services. Exception faite des cas plus originaux, par exemple, dans le domaine de la mode, où les petites entreprises offrent des services de location de vêtements [RAE 17] [STAL 17].

Les stratégies axées sur la création de valeur circulaire à partir de la valorisation des matériaux (symbiose industrielle et recyclage) sont très minoritaires dans l'ensemble des articles étudiés. L'extension de la valeur des ressources ou de l'utilisation des sous-produits et des déchets se produit dans les cas relatifs au secteur agricole (voir Devisscher \& Mont [DEV 08], Pereira, CarballoPenela, González-López, Vence [PER 16a] et sur une base minoritaire dans la fabrication de produits dont la propriété est conservée par les fabricants et dont la valeur de fin de vie est élevée ou facilement récupérable [BRE 18] [LIN 14] [YAN 18].

En ce qui concerne l'impact environnemental des SPS analysés dans les différents articles, il est difficile de tirer une conclusion générale, pour plusieurs raisons : premièrement, il n'existe pas de méthodologie unique pour réaliser cette analyse. Ainsi, très peu utilisent des méthodologies du type mesure de l'empreinte carbone ou analyse du cycle de vie, alors que la plupart font des évaluations qualitatives. Deuxièmement, plusieurs articles analysent des cas hypothétiques, de sorte que les analyses sont fondées sur des estimations. Les cas réels présentent également des difficultés de mesure, en fonction des limites établies (produit, service, chaîne de valeur, etc.). Troisièmement, il est également important de souligner la difficile comparabilité, en raison de la portée et des limites différentes de chaque cas : il n'est pas identique d'analyser l'impact sur l'efficacité de la collecte des déchets urbains d'un outil technologique donné ou l'économie de produits chimiques dans une technologie de nettoyage des bâtiments [LIN 14], que l'impact de la combinaison de différents types de SPS sur l'ensemble de la chaîne de production d'un produit alimentaire tel que le café [DEV 08].

Une autre question pertinente au débat sur le potentiel du SPS en tant que business model pour l'économie circulaire est le sens dans lequel la relation se déroule. En d'autres termes, la conception 
et la mise en œuvre des SPS sont-elles un système organisationnel d'activités qui facilite l'adoption de stratégies d'économie circulaire ou, inversement, est-ce l'adoption de stratégies pour l'économie circulaire qui exige de nouveaux business models, qui dans certains cas peuvent être classés comme SPS ? Dans plusieurs des articles examinés, comme dans la documentation sur les SPS et la servitisation en général, l'accent est mis sur le statut des produits. En particulier, les avantages environnementaux des SPS sont attribués au fait que les fabricants en demeurent propriétaires. Toutefois, l'adoption de ces modèles, ou le passage de la vente de produits purs à des modèles de services avancés, n'est pas toujours réalisable ou souhaitable pour les entreprises manufacturières [EME 10] [GEB 13] [HEL 16] [KOW 15] [NEE 08] [PAG 17] [PAI 13] [VAL 17] [VIN 16].

Les exemples analysés dans cet article montrent également qu'il est très rare qu'une entreprise assume l'entière responsabilité du bouclage des cycles. En ce sens, la littérature sur les business models pour l'économie circulaire souligne l'importance d'établir de nouvelles relations de collaboration, de créer des réseaux de business models, pour contribuer à la circularité [ANT 16] [BSI 17]. Par conséquent, la clé de la circularité ne serait pas tant la conservation de la propriété du produit. Le point de départ devrait être le respect des principes de l'EC et la conception des modèles d'entreprise les plus appropriés pour sa mise en œuvre dans la pratique et d'un point de vue systémique.

Un autre aspect discutable est l'importance accordée dans la literature à la servitisation et aux SPS axés sur les résultats et, dans une moindre mesure, aux SPS axés sur l'usage. En admettant l'hypothèse de Spring \& Araujo [SPR 17] et en identifiant les possibilités des nouveaux services conformément à la proposition de la «Value Hill »[ACH 16], l'économie circulaire pourrait bénéficier d'une amélioration des services traditionnels ou de nouveaux services associés aux produits. En ce sens, ces services sont généralement fournis dans les SPS mentionnés, puisque les fabricants qui conservent la propriété et fournissent un service sont motivés à exercer ce type d'activité (réparation, rénovation, refabrication) plutôt qu'à acheter un nouveau produit (ce ne serait pas rentable puisqu'il s'agit de produits qui sont généralement très chers). Toutefois, cette hypothèse doit faire l'objet d'une étude empirique supplémentaire, car dans certains secteurs, le prestataire de services peut être en mesure d'imposer des prix élevés en échange du remplacement rapide d'un bien par un autre (comme c'est le cas, par exemple, des sociétés de location de voitures qui renouvellent leurs véhicules tous les $2 / 3$ ans).

D'autre part, étant donné que tous les produits ne sont pas adaptés à une utilisation partagée ou à la distribution d'un résultat ou d'une fonctionnalité, en particulier dans certains segments de produits de consommation, les services de réparation, d'entretien, de mise à niveau et de renouvellement devraient être mis en valeur dans la transition vers l'économie circulaire. Il arrive souvent que ces services soient aujourd'hui marginalisés parce qu'il s'agit d'activités à forte intensité de main-d'œuvre et en raison de la complexité croissante des produits, les nouveaux achats sont encouragés plutôt que la réparation des produits.

En résumé, d'après les cas analysés dans la literature, il semble que, en général, les SPS ont le potentiel de contribuer à la réalisation de l'économie circulaire. Cependant, la réalisation de ce potentiel n'est pas automatique, mais dépend de la stratégie et du business model d'ensemble dans lequel il est mis en œuvre. L'examen de la littérature ne permet pas non plus de tirer des conclusions claires quant à la pertinence d'un type de SPS ou d'un autre. En effet, des études de cas montrent que la contribution à la réalisation des objectifs de l'EC ne dépend pas seulement d'un produit-service spécifique mais d'une combinaison créative de différents services, en fonction de la nature du produit et de l'utilisateur (que ce soit pour d'autres entreprises ou pour les consommateurs finaux) et même de l'intégration de smart technologies offertes par des entreprises technologiques (e.g., optimisation des activitées logistiques par des conexions machine-to-machine, comme le montrent 
les cas analysés par Lelah et al [LEL 11] et Lindström et al [LINDS 17]). C'est l'adoption de stratégies spécifiques, en l'occurrence liées à différents business models, qui peut contribuer à l'économie circulaire.

Le SPS peut être un instrument d'une stratégie d'économie circulaire et durable, mais il peut aussi rester un business model linéaire axé sur la maîtrise de l'ensemble de la chaîne par le fournisseur, la fidélisation de la clientèle et la maîtrise des connaissances et du savoir-faire de la phase d'utilisation de la marchandise. Ceci est particulièrement pertinent dans le cas de la fourniture d'équipements et de technologies pour les services publics (santé, transports, infrastructures technologiques, services municipaux, etc.), puisque dans ces cas le SPS peut même constituer, le cas échéant, une modalité ou un moyen de privatisation des services publics.

\section{SPS Circulaire ou stratégie commerciale: le cas de Philips}

Philips a lancé son programme d'économie circulaire en 2012, en le liant clairement aux objectifs de compétitivité de l'entreprise : "Dans une économie circulaire, l'utilisation plus efficace des matériaux permet de créer plus de valeur, à la fois par la réduction des coûts et par le développement de nouveaux marchés ou la croissance des marchés existants". A cette fin, l'entreprise a l'intention de clore complètement le cycle pour les gros équipements médicaux en 2020- par exemple l'imagerie par résonance magnétique - par la reprise de ces équipements.

En 2017, Philips a tiré $11 \%$ de ses revenus des produits et services du programme d'économie circulaire; son objectif est d'atteindre $15 \%$ d'ici 2020. Jusqu'à présent, l'entreprise applique à titre expérimental des modèles basés sur l'accès et la fourniture de solutions. En particulier, dans le domaine des grands équipements médicaux et de santé, Philips a lancé le service de mise à niveau et de rachat SmartPath, qui vise à prolonger la durée de vie utile des équipements médicaux (par exemple, résonance magnétique, radiographie, ultrasons, etc.), offrant les avantages suivants:

* La mise à jour et l'amélioration continue de l'équipement, en maintenant la qualité et la performance de l'équipement sur le long terme;

* Ajoutez de nouveaux services/fonctionnalités qui permettent aux clients (fournisseurs de services médicaux et de santé) de créer de nouvelles lignes de services et de nouvelles sources de revenus;

* Prolonger la durée de vie de l'équipement;

* Échanger des équipements désuets contre des équipements neufs ou remis à neuf.

Un autre programme Philips est Technology Maximizer, qui fait partie de la proposition de valeur pour mettre à jour efficacement le logiciel de l'équipement. Il convient toutefois de noter que la société elle-même déclare que l'étendue et la disponibilité des mises à niveau seront déterminées par Philips et peuvent varier d'un système à l'autre.

Le message clé de Philips pour ce type de services est que le client évitera les problèmes dans les services de santé, en s'assurant qu'il dispose d'équipements et de pièces de rechange à jour, d'un personnel spécialisé, qu'il se tient au courant des progrès constants de la technologie d'imagerie et qu'il évite ainsi les coûts de transaction découlant de l'embauche individuelle de chacun. D'autre part, il est clair que cette stratégie a pour objectif ultime le contrôle direct de l'ensemble de la chaîne de valeur jusqu'à la relation directe avec le client, ce qui confère à l'entreprise une position privilégiée sur ce marché. En fait, au cours du H2020 projet R2PI 
(www.r2piproject.eu), qui analyse le programme SmartPath de Philips, il a été constaté que les clients (prestataires de soins de santé) sont toujours réticents à des services tels que SmartPath en raison, justement, de la possibilité de perdre le contrôle sur le processus d'obtention d'images pour le diagnostic.

De plus, lorsqu'elle s'applique à la privatisation en santé publique, c'est une voie qui peut permettre la privatisation progressive du service de diagnostic sanitaire. C'est précisément ce qui se passe avec certains programmes de marchés publics innovants entre les services de santé publique espagnole et les entreprises prestataires de SPS (Programme H2050 et Innova Saúde de Sergas-Galicia ou Código100 du Ministère Espagnol des Sciences).

\section{Conclusion}

Dans cet article, nous avons réalisé une revue de la littérature, en essayant de réfléchir de façon critique à la contribution des SPS à la réalisation de l'économie circulaire. Avec l'éco-innovation, les nouveaux business models devraient constituer l'un des piliers de la transition des économies vers le nouveau paradigme de production et de consommation de l'économie circulaire. Parmi ces modèles d'affaires, au cours des dernières années, les SPS ont attiré beaucoup d'attention de la part des universitaires et des consultants. Les SPS consistent en un panier combiné de produits et de services, capable de satisfaire ensemble les besoins des clients, qu'ils soient intermédiaires (autres organisations) ou consommateurs finaux. La stratégie d'incorporation de services dans les produits est née comme une tendance parmi les entreprises manufacturières, dans le but de se différencier et d'obtenir des avantages concurrentiels sur les marchés matures. Par la suite, on a commencé à analyser la possibilité que les SPS contribuent à une meilleure performance environnementale, en se basant sur deux prémisses : premièrement, l'immatérialité supposée des services et la possibilité de satisfaire les besoins des clients sur la base des fonctions des produits plutôt que sur les produits eux-mêmes ; deuxièmement, la conservation de la propriété des biens par les fournisseurs, assumant ainsi la responsabilité de leur performance non seulement pendant la phase de production mais aussi pendant la phase d'utilisation et jusqu'à leur fin de vie (gérée par les consommateurs dans des modèles commerciaux traditionnels). Cependant, jusqu'à présent, la littérature qui étudie la contribution réelle de la SPS à l'EC est très limitée. Les articles examinés dans le cadre de nos travaux nous permettent de conclure qu'il n'y a aucune garantie que les SPS contribueront automatiquement à l'atteinte des principes de l'EC.

La conclusion la plus générale que l'on peut tirer à partir des cas étudiés est qu'il est nécessaire d'adopter une vision critique et prudente avant d'admettre que les SPS mènent généralement à une meilleure performance environnementale. Bien qu'il existe des preuves d'une utilisation plus efficace des ressources grâce à des SPS axés sur les produits et l'utilisation, les cas réels de SPS fondés sur les résultats sont à peine analysés. En ce sens, il convient d'éviter l'utilisation abusive en termes de marketing ou de ressources publiques, d'une supposée identification automatique entre SPS et EC ou entre SPS et soutenabilité.

Pour que les SPS contribuent réellement à l'EC et à la durabilité, ils doivent être intégrés dans un changement systémique plus large. Par exemple, la prise en charge de la responsabilité individuelle élargie du producteur/fournisseur peut mener à des incitations pour une utilisation plus efficace des produits et de leurs composants par le biais de stratégies de réutilisation, de réparation, de mise à niveau, de reconditionnement, de ré-usinage et de recyclage. Il faut également une infrastructure de récupération et un cadre réglementaire qui permette et encourage la réutilisation.

Finalement, pour que les SPS contribuent à la transition vers un nouveau paradigme tel que proposé théoriquement par l'économie circulaire, il est nécessaire d'intégrer des changements 
institutionnels, fiscaux et réglementaires qui redéfinissent les critères de valeur des entreprises et des consommateurs. En d'autres termes, toute la chaîne doit être repensée, depuis la conception jusqu'aux modes d'utilisation/consommation. Dans ce contexte, l'économie de la fonctionnalité acquiert un sens beaucoup plus large et ambitieux pour progresser vers les objectifs de l'économie circulaire.

\section{Bibliographie}

[ACH 16] ACHTERBerg E., HinfelaAR J., BocKen N., The Value Hill Business Model Tool: identifying gaps and $\begin{array}{llllll}\text { opportunities in } & \text { a } & \text { network, } & \text { Retrieved } & \text { from }\end{array}$ http://www.scienceandtheenergychallenge.nl/sites/default/files/multimedia/organization/sec/2016-0616_NWO_Sc4CE/NWO Sc4CE - Workshop Business Models - Paper on Circular Business Models.pdf

[ANT 16] ANTIKAinen M., ValKoKARi K., MCClelland J., “A framework for sustainable circular business model innovation”, Technology Innovation Management Review, 6(7), p. 5-12, 2016.

[BAi 07] Baines T. S., Lightfoot H. W., Evans S., Neely A, Greenough R., PePPard J., Wilson H., "State-ofthe-art in product-service systems", Proceedings of the Institution of Mechanical Engineers, Part B: Journal of Engineering Manufacture, 221(10), 1543-1552, 2007. http://doi.org/10.1243/09544054JEM858

[BAR 03] Bartolomeo M., Dal Maso, D., De Jong P., Eder P., Groenewegen P., Hopkinson P., Zaring O., "Eco-efficient producer services-what are they, how do they benefit customers and the environment and how likely are they to develop and be extensively utilised?", Journal of Cleaner Production, 11(8), p. 829-837, 2003, http://doi.org/10.1016/S0959-6526(02)00157-9

[BeU 13] Beuren F. H., Gomes Ferreira M. G., CAuchick Miguel P. A. (2013). «Product-service systems: a literature review on integrated products and services", Journal of Cleaner Production, 47, p. 222-231, 2013, http://doi.org/10.1016/j.jclepro.2012.12.028

[BOC 16] Bocken N. M. P. N., BAKKer C., DE PAuW I., BAKKer C., VAN DER GRinTen B., "Product design and business model strategies for a circular economy", Issue 5: Sustainable Design and Manufacturing for Circular Economy, Journal of Industrial and Production Engineering, 33(5), p. 12-14, 2016. http://doi.org/10.1080/21681015.2016.1172124

[BRE 18] Bressanelli G., Adrodegari F., Perona M., Saccani N., "Exploring how usage-focused business models enable circular economy through digital technologies", Sustainability (Switzerland), 10(3), 2018, http://doi.org/10.3390/su10030639

[BSI 17] BSI Standards Publication, Framework for implementing the principles of the circular economy in organizations - Guide, 2017.

[CES 13] CESCHIN F, "Critical factors for implementing and diffusing sustainable product-Service systems: insights from innovation studies and companies' experiences", Journal of Cleaner Production, 45(0), p. 74-88, 2013, http://doi.org/http://dx.doi.org/10.1016/j.jclepro.2012.05.034

[CHE 11] CHESBROUGH H., Open services innovation (Galician T), Barcelona: Plataforma Editorial, 2011.

[DEV 08] Devisscher T., MONT O., "An analysis of a product service system in Bolivia: coffee in Yungas", International Journal of Innovation and Sustainable Development, 3, p. 262-284, 2008.

[EME 10] Emerald Group Publishing, "Challenges in transforming manufacturing organisations into product-service providers", Journal of Manufacturing Technology Management, 21(4), p. 449-469, 2010.

[EMF 13] EMF, Towards the circular economy. Economic and business rationale for an accelerated transition, 2013, Retrieved from https://www.ellenmacarthurfoundation.org/publications.

[EPA 09] EPA, "Green Servicizing” for a more sustainable US Economy. Key concepts, tools and analyses to inform policy engagement, 2009, Retrieved from http://www.epa.gov/wastes/conserve/tools/stewardship/docs/greenservice.pdf

[EVA 07] EVANS S., PARTIDÁRIO P. J., LAMBERT J., "Industrialization as a key element of sustainable product-service solutions", International Journal of Production Research, 45(18-19), p. 4225-4246, 2007. http://doi.org/10.1080/00207540701449999 
[FUR 10] FURRER O., "A customer relationship typology of product services strategies”, dans F. Gallouj, F. Djellal (Eds.), The handbook of innovation and services: a multi-disciplinary perspective, Cheltemham: Edward Elgar Publishing Limited, p. 701-721, 2010.

[GEB 13] Gebauer H., Paiola M., SACCANi N., «Characterizing service networks for moving from products to solutions", Industrial Marketing Management, 42(1, SI), p. 31-46, 2013, http://doi.org/10.1016/j.indmarman.2012.11.002

[GEI 17] Geissdoerfer M., Savaget P., Bocken N. M. P., Hultink E. J., "The Circular Economy - A new sustainability paradigm?", Journal of Cleaner Production, 143, p. 757-768, 2017, http://doi.org/10.1016/j.jclepro.2016.12.048

[GHI 14] Ghisellini P., Cialani C., Ulgiati S., "A review on circular economy: the expected transition to a balanced interplay of environmental and economic systems", Journal of Cleaner Production, 114, p. 11-32, 2014, http://doi.org/10.1016/j.jclepro.2015.09.007

[GOE 99] Goedkoop M. J., Van Halen C. J. G., Te Riele H. R. M., Rommens P. J. M., Product Service systems, Ecological and Economic Basics, Report for the Dutch ministries of Environment (VROM) and Economic Affairs (EZ). The Hague, 1999, Retrieved from http://teclim.ufba.br/jsf/indicadores/holan Product Service Systems main report.pdf

[Hal 04] Halme M., Hrauda G., Jasch C., Jonuschat H., Kortman J., Trindade P., Velte D., SUSTAINABLE HOMESERVICES Benchmarking Sustainable Services for the Housing Sector in the City of Tomorrow, Scientific Report to the European Union, 2004.

[HEL 16] HeLmS T., "Asset transformation and the challenges to servitize a utility business model", Energy Policy, 91, p. 98-112, 2016, http://doi.org/10.1016/j.enpol.2015.12.046

[HUI 16] Huikkola T., KohtamäKi M., Rabetino R., "Resource realignment in servitization", ResearchTechnology Management, 59(4), p. 30-39, 2016, http://doi.org/10.1080/08956308.2016.1185341

[JOH 16] Johansson C., Elfsberg J., LARSSON T. C., Frank M., LeIFER L. J., NiLSSON N., SÖDERBERG V., Urban Mining as a Case for PS,. Procedia CIRP, 47, 460-465, 2016. http://doi.org/10.1016/j.procir.2016.03.089

[KOH 18] KohtamÄKi M., Baines T., Rabetino R., Bigdeli A. Z., Practices and tools for servitization. Managing service transition, Palgrave Macmillan, 2018. http://doi.org/https://doi.org/10.1007/978-3-319-76517-4

[KOM 05] Комото H., Tomiyama T., Nagel M., Silvester S., Brezet H., "Life Cycle Simulation for analyzing Product Service Systems", in Environmentally Conscious Design and Inverse Manufacturing, 2005. Eco Design 2005. Fourth International Symposium on, p. 386-393, 2005. Tokyo: IEEE. http://doi.org/10.1109/ECODIM.2005.1619251

[KOW 15] KowalKowski C., Windahl C., KindSTRÖM D., GebaUER H., "What service transition? Rethinking established assumptions about manufacturers' service-led growth strategies", Industrial Marketing Management, 45(November), 59-69, 2015, http://doi.org/10.1016/j.indmarman.2015.02.016

[LEE 12] LEE S., GeUM Y., LEE H., PARK Y., "Dynamic and multidimensional measurement of product-service system (PSS) sustainability: a triple bottom line (TBL)-based system dynamics approach", Journal of Cleaner Production, 32, p. 173-182, 2012, http://doi.org/10.1016/j.jclepro.2012.03.032

[LEL 11] Lelah A., MathieuX F., BRISSAud D., "Contributions to eco-design of machine-to-machine product service systems: The example of waste glass collection", Journal of Cleaner Production, 19(9-10), p. 1033-1044, 2011, http://doi.org/10.1016/j.jclepro.2011.02.003

[LIN 14] Lindahl M., Sundin E., SaKaO T., "Environmental and economic benefits of Integrated Product Service Offerings quantified with real business cases", Journal of Cleaner Production, 64, p. 288-296, 2014, http://doi.org/10.1016/j.jclepro.2013.07.047

[LINDE 17] LiNDER M., WILliander M., "Circular Business Model Innovation: Inherent Uncertainties", Business Strategy and the Environment, 26(2), p. 182-196, 2017, http://doi.org/10.1002/bse.1906

[LINDS 17] Lindström J., HeRmanson A., Hellis M., KYösti P., Optimizing Recycling Management Using Industrial Internet Supporting Circular Economy: A Case Study of an Emerging IPS2. Procedia CIRP, 64, 55-60, 2017, http://doi.org/10.1016/j.procir.2017.03.072 
[MER 18] Merli R., Preziosi M., ACAMPORA A., "How do scholars approach the circular economy? A systematic literature review", Journal of Cleaner Production, 178, p. 703-722, 2018, http://doi.org/10.1016/j.jclepro.2017.12.112

[MiC 17] Michelini G., Moraes R.N., Cunha R.N., Costa J.M.H., OMetto A.R., From Linear to Circular Economy: PSS Conducting the Transition, Procedia CIRP, 64, 2-6., 2017, https://doi.org/10.1016/j.procir.2017.03.012

[MON 02a] MonT O., "Clarifying the concept of product-service system", Journal of Cleaner Production, 10(3), p. 237-245, 2002a, http://doi.org/10.1016/S0959-6526(01)00039-7

[MON 02b] MONT O., "Drivers and barriers for shifting towards more service-oriented businesses : Analysis of the PSS field and contributions from Sweden", The Journal of Sustainable Product Design, 2, p. 89-103, 2002b.

[NEE 08] NEELY A., "Exploring the financial consequences of the servitization of manufacturing", Operations Management Research, 1(2), p. 103-118, 2008. http://doi.org/10.1007/s12063-009-0015-5

[OLI 03] Oliva R., KAllenBerg R., "Managing the transition from products to services", International Journal of Service Industry Management, 14(2), p. 160-172, 2003. http://doi.org/10.1108/09564230310474138

[OMA 03] OMAnN, I., "Product Service Systems and their Impacts on Sustainable Development. A multi-criteria evaluation for Austrian companies", Frontiers, p. 1-34, 2003.

[OST 10] OSTERWalder A., Pigneur Y., Business model generation: a handbook for visionaries, game changers, and challengers, John Wiley \& Sons, 2010.

[OST 05] OSTERWALDER A., PIGNEUR Y., TUCCI C. L., "Clarifying business models: Origins, present, and future of the concept", in Communications of the association for Information Systems, Vol. 16, 2005, Retrieved from http://aisel.aisnet.org/cgi/viewcontent.cgi?article=3016\&context=cais

[PAG 17] Pagoropoulos A., Laumann KJer, L., AXel Bejbro Andersen, J., MCAloone, T. C., "The influence of costs and benefits' analysis on service strategy formulation: Learnings from the shipping industry", Cogent Engineering, 4(1), p. 1-20, 2017, http://doi.org/10.1080/23311916.2017.1328792

[PAi 13] Paiola M., Saccani N., Perona M., Gebauer H., "Moving from products to solutions: Strategic approaches for developing capabilities", European Management Journal, 31(4), p. 390-409, 2013, http://doi.org/10.1016/j.emj.2012.10.002

[PER 16a] Pereira Á., CARballo-Penela A., GonZÁlez-LÓPEZ M., VENCE X., "A case study of servicizing in the farming-livestock sector: organisational change and potential environmental improvement", Journal of Cleaner Production, 124, p. 84-93, 2016, http://doi.org/10.1016/j.jclepro.2016.02.127

[PER 17] Pereira A., TURnes A., Vence X., "Barriers to shifting to a servicized model of crop protection in smallholding viticulture", Journal of Cleaner Production, 149, p. 701-710, 2017. http://doi.org/10.1016/j.jclepro.2017.02.126

[PER 16b] PEREIRA Á., VENCE X., Terciarización, servizalización e sustentabilidade. Un novo paradigma produtivo, Andavira, 2016.

[RAB 16] RABETINO R., KOHTAMÄKi M., GEBAUER H., "Strategy map of servitization", International Journal of Production Economics, (in press), 2016.

[RAE 17] RÆBILD U., BANG A. L., "Rethinking the Fashion Collection as a Design Strategic Tool in a Circular Economy”, The Design Journal, 20(sup1), p. S589-S599, 2017, http://doi.org/10.1080/14606925.2017.1353007

[REI 14] REIM W., PARIDA V., ÖRTQVIST D., "Product-Service Systems (PSS) business models and tactics - a systematic literature review", Journal of Cleaner Production, 2014. http://doi.org/10.1016/j.jclepro.2014.07.003

[ROT 07] Rothenberg S., "Sustainability through servicizing", MIT Sloan Management Review, 48(2), p. 82-91, 2007.

[ROY 00] ROY R., "Sustainable product-service systems”, Futures, 32(3-4), p. 289-299, 2000, http://doi.org/10.1016/S0016-3287(99)00098-1

[SPR 17] SPRING M., ARAUJO L., "Product biographies in servitization and the circular economy", Industrial Marketing Management, 60, p. 126-137, 2017, http://doi.org/10.1016/j.indmarman.2016.07.001 
[STAH 97] STAHEL W. R., "The service economy:'wealth without resource consumption'? Philosophical Transactions of the Royal Society of London A: Mathematical, Physical and Engineering Sciences, 355(1728), p. 1309-1319, 1997.

[STA 16] STAHEL W. R., “Circular Economy”, Nature, 531, p. 435-438, 2016. http://doi.org/10.1038/531435a

[STAL 17] STÅL H. I., JANSSON J., "Sustainable Consumption and Value Propositions: Exploring Product-Service System Practices Among Swedish Fashion Firms", Sustainable Development, 25(6), p. 546-558, 2017, http://doi.org/10.1002/sd.1677

[TOF 02] TOFFEL M. W., "Contracting for Servicizing", SSRN Electronic Journal, n ${ }^{\circ}$ 8-63, 2002, Retrieved from http://www.ssrn.com/abstract=1090237

[TUK 04] TUKKER A., "Eight types of product-service system: eight ways to sustainability? Experiences from SusProNet", Business Strategy and the Environment, 13(4), p. 246-260, 2004. http://doi.org/10.1002/bse.414

[TUK 15] TUKKER A., "Product services for a resource-efficient and circular economy - A review", Journal of Cleaner Production, 97, p. 76-91, 2015. http://doi.org/10.1016/j.jclepro.2013.11.049

[TUK 06] TUKKER A., TISCHNER U., "Product-services as a research field: past, present and future. Reflections from a decade of research", Journal of Cleaner Production, 14(17), p. 1552-1556, 2006, http://doi.org/10.1016/j.jclepro.2006.01.022

[URB 17] URbinati A., Chiaroni D., ChiESA V., "Towards a new taxonomy of circular economy business models", Journal of Cleaner Production, 167, p. 487-498, 2017, doi: 10.1016/j.jclepro.2017.09.047.

[VAL 17] VALTAKOSKI A., "Explaining servitization failure and deservitization: A knowledge-based perspective", Industrial Marketing Management, 60, p. 138-150, 2017. http://doi.org/10.1016/j.indmarman.2016.04.009

[VAN 13] Van Ostaeyen J., Van Horenbeek A., Pintelon L., Duflou J. R., "A refined typology of productservice systems based on functional hierarchy modelling", Journal of Cleaner Production, 51, p. 261-276, 2013. http://doi.org/10.1016/j.jclepro.2013.01.036

[VAN 88] VANDERMERWE S., RADA J., "Servitization of business: adding value by adding services", European Management Journal, 6(4), p. 314-324, 1988. http://doi.org/10.1016/0263-2373(88)90033-3

[VEN 19] Vence, X., PereIRA Á., « Eco-innovation and Circular Business Models as drivers for a circular economy”, Contaduría y Administración, 64(1), p. 1-19, 2019.

[VIS 15] VISNJIC I., ARTS S., RINGOV D., How do industry evolution and industry conditions prompt product firms to offer services? Cambridge Service Alliance, 2015, Retrieved from http://cambridgeservicealliance.eng.cam.ac.uk/resources/Downloads/Monthly Papers/2015OctoberPaper_IndustryEvolutionandConditions.pdf

[VIS 16] Visnjic I., Wiengarten F., Neely A., "Only the Brave: Product Innovation, Service Business Model Innovation, and Their Impact on Performance", Journal of Product Innovation Management, 33(1), p. 36-52, 2016. http://doi.org/10.1111/jpim.12254

[VIS 13] ViSNJIC I, KASTALLI I., VAN LOOY, B., "Servitization: Disentangling the impact of service business model innovation on manufacturing firm performance", Journal of Operations Management, 31(4), p. 169-180, 2013. http://doi.org/10.1016/j.jom.2013.02.001

[Whi 99] White A. L., Stoughton M., Feng L., Servicizing: The quiet transition to Extended Product Responsibility, 1999. Retrieved from http://infohouse.p2ric.org/ref/17/16433.pdf

[YAN 18] Yang M., Smart P., Kumar M., Jolly M., Evans S., "Product-service systems business models for circular supply chains", Production Planning and Control, 29(6), p. 1366-5871, 2018, http://doi.org/10.1080/09537287.2018.1449247 